\title{
ON THE COMPLEX OSCILLATION OF NON-HOMOGENEOUS LINEAR DIFFERENTIAL EQUATIONS WITH MEROMORPHIC COEFFICIENTS
}

\author{
By CHEN ZONG-XUAN and GAO SHI-AN
}

\begin{abstract}
In this paper, we investigate the complex oscillation of

$$
f^{(k)}+b_{k-1} f^{(k-1)}+\cdots+b_{0} f=B(z),
$$

where $b_{k-j}(\jmath=1, \cdots, k)$ are rational functions, $B(z)$ is a meromorphic funciton, and obtain general estimates of the exponent of convergence of the zerosequence and the pole-sequence of solutions for the above equation.
\end{abstract}

Key words: Non-homogeneous Linear differential equation, Meromorphic function, zero-sequence, Pole-sequence, Exponent of convergence.

\section{$\S 1$. introduction and results.}

For convenience in our statement, we first explain the notations used in this paper, we will use resperctively the notations $\lambda(f)$ and $\lambda(1 / f)$ to denote the exponent of convergence of the zero-sequence and the pole-sequence of a meromorphic function $f(z), \bar{\lambda}(f)$ and $\bar{\lambda}(1 / f)$ to denote the exponent of convergence of the sequences of distinct zeros and distinct poles of $f(z), \sigma(f)$ to denote the order of growth of $f(z), \Upsilon_{f}(r)$ to denote the centralindex of entire function $f(z)$. By the Wiman-Valiron theory, we have $\sigma(f)=\varlimsup_{\gamma \rightarrow \infty} \frac{\log \Upsilon_{f}(r)}{\log r}$.

In addition, other notations of the Nevanlinna theory are standard (e.g. see [3]), the individual ones will be shown when they appear.

We also need the following Definition.

DEFINITION. If the meromorphic function $f(z)$ has infinitely many zeros, we call $f(z)$ is oscillatory.

The complex oscillatory problems of the non-homogeneous linear differential equation

\footnotetext{
* Project supported by the National Natural Science Foundation of China.

Received April 2, 1991.
} 


$$
f^{(k)}+a_{k-1} f^{(k-1)}+\cdots+a_{0} f=F
$$

are a very important aspect in the complex oscillation theory of differential equations which has been an active research area recently. Just lately, Gao Shi-an proved in [2].

THEOREM A. Let $F$ be a transcedental entire function with $\sigma(F)<\infty, a_{k-1}$ $(j=1, \cdots, k)$ polynomials. Then for every solution $f$ of

$$
f^{(k)}+a_{k-1} f^{(k-1)}+\cdots+a_{0} f=F \quad(k \geqq 1)
$$

(a) If $F$ is oscillatory, then $f$ is also oscillatory.

(b) $\lambda(f) \geqq \lambda(F)$.

(c) If $\sigma(F)$ is not a positive integer, then

$$
\lambda(f)=\sigma(f) \geqq \sigma(F)=\lambda(F) .
$$

(d) If $\sigma(f)>\sigma(F)$, then $\lambda(f)=\sigma(f)>\sigma(F)$.

THEOREM B. For the equation

$$
f^{\prime \prime}+a_{0} f=P_{1} e^{p_{0}}
$$

where $a_{0}, p_{0}, p_{1}$ are polynomials, $\operatorname{deg} a_{0}=n, \operatorname{deg} p_{0}<1+(n / 2)$.

(a) If $n>1$ and $\operatorname{deg} P_{1}<n$, then every solution $f$ of (1.2) satisfies

$$
\lambda(f)=\bar{\lambda}(f)=\sigma(f)=1+\frac{n}{2}>\operatorname{deg} P_{0} .
$$

(b) If $\operatorname{deg} p_{1} \geqq n \geqq 0$, then the solution $f$ of (1.2) either satisfies $\lambda(f)=\bar{\lambda}(f)$ $=\sigma(f)=1+(n / 2)>\operatorname{deg} P_{0}$, or is of the form $f=Q e^{P_{0}}$, where $Q$ is a polynomial. And if (1.2) has a solution of the form $Q e^{P_{0}}$ with $Q$ polynomial, then (1.2) must have solutions which satisfy $\lambda(f)=\bar{\lambda}(f)=\sigma(f)=1+(n / 2)>\operatorname{deg} P_{0}$.

In this paper, we investigate the complex oscillatin of non-homogeneous linear differential equations with meromorphic coefficients, and obtain general estimates of the exponent of convergence of the zero-sequence and the polesequence of solutions for the considered equations.

In fact, we will prove the following theorems in this paper.

THEOREM 1. Let $A \neq 0, b_{k-j}(\jmath=1, \cdots, k)$ be rational functions, $b_{k-\jmath}$, have a pole at $\infty$ of order $n_{k-\jmath} \geqq 0, k \geqq 1, P$ be a polynomial $\operatorname{deg} P=\beta$ satisfies

$$
1+\max _{1 \leq \jmath \leq k} \frac{n_{k-\jmath}}{\jmath}<\beta<\infty .
$$

If the differential equation

$$
f^{(k)}+b_{k-1} f^{(k-1)}+\cdots+b_{0} f=A e^{P}
$$


has a meromorphic solution $f$, then

(a) $\sigma(f)=\beta, f$ has only finitely many poles.

(b) suppose that $A$ has a pole at $\infty$ of order $n_{A}$. If $n_{A}<k(\beta-1)$, then $\bar{\lambda}(f)$ $=\lambda(f)=\sigma(f)=\beta$, if $n_{A} \geqq k(\beta-1)$, then all meromorphic solutions of (1.4) satisfy $\bar{\lambda}(f)=\lambda(f)=\sigma(f)=\beta$, except at most a possible one. The possible exceptional one is of the form $f_{0}=A_{0} e^{P}$ ( $A_{0}$ is rational).

TheOREM 2. Let $A \neq 0, b_{k-j}(j=1, \cdots, k)$ be rational functions, $b_{k-\jmath}$ have a pole at $\infty$ order $n_{k-\jmath} \geqq 0, k \geqq 1, P$ be a polynomial, $\operatorname{deg} P=\beta \leqq 1+\max _{1 \leqq \jmath \leqq k} \frac{n_{k-\jmath}}{\jmath}$. If (1.4) has a meromorphic solution $f$, then

(a) $\beta \leqq \sigma(f) \leqq 1+\max _{1 \leqq j \leqq k} \frac{n_{k-\jmath}}{j}$, $f$ has only finitely many poles.

(b) If $\sigma(f)>\beta$, then $\bar{\lambda}(f)=\lambda(f)=\sigma(f)$.

TheOREM 3. Let $b_{k-j}(j=1, \cdots, k)$ be rational functions having a pole at $\infty$ of order $n_{k-\jmath} \geqq 0, k \geqq 1, B(z)$ be a transcendental meromorphic function, $\sigma(B)=\beta$ satisfying (1.3). If all solutions of the differential equation

$$
f^{(k)}+b_{k-\jmath} f^{(k-1)}+\cdots+b_{0} f=B(z)
$$

are meromorphic functions, then

(a) $\sigma(f)=\beta$.

(b) $\lambda(1 / f)=\lambda(1 / B), \bar{\lambda}(1 / f)=\bar{\lambda}(1 / B)$. If $\lambda(B)>\lambda(1 / B)$, then $\lambda(f) \geqq \lambda(B)$.

(c) If $\beta>\max \{\lambda(B), \lambda(1 / B)\}$, then all solutions of (1.5) satisfy $\bar{\lambda}(f)=\lambda(f)=\sigma$ $(f)=\beta$, except at most a possible one. The possible exceptional one $f_{0}$ satisfies $\lambda\left(f_{0}\right)<\beta$.

THEOREM 4. Let $b_{k-j}(j=1, \cdots, k)$ be rational functions having a pole at $\infty$ of order $n_{k-\jmath} \geqq 0, k \geqq 1, B(z) \neq 0$ be a meromorphic function satisfying $\sigma(B)=\beta$ $\leqq 1+\max _{1 \leqq \jmath \leqq k} \frac{n_{k-\jmath}}{\jmath}$. If all solutions of (1.5) are meromorphic functions, then

(a) $\beta \leqq \sigma(f) \leqq 1+\max _{1 \leqq \jmath \leqq k} \frac{n_{k-\jmath}}{j}$.

(b) $\lambda(1 / f)=\lambda(1 / B), \bar{\lambda}(1 / f)=\bar{\lambda}(1 / B)$. If $\lambda(B)>\lambda(1 / B)$, then $\lambda(f) \geqq \lambda(B)$.

(c) If $\sigma(f)>\beta$, then $\bar{\lambda}(f)=\lambda(f)=\sigma(f)$.

\section{§2. Lemmas.}

LEMMA 1. Let the set $E \leqq[0,+\infty)$ have finite logarithmic measure, $Y(r)$ be a nondecreasing function on $[0, \infty)$. Then

$$
\varlimsup_{\substack{r \rightarrow \infty \\ r \in[0,+\infty)}} \frac{\log \gamma(r)}{\log r}=\varlimsup_{\substack{r \in[0,+\infty)-E \\ r \rightarrow \infty}} \frac{\log r(r)}{\log r} .
$$


Proof. We clearly have

$$
\varlimsup_{\substack{r \rightarrow \infty \\ r \in[0,+\infty)}} \frac{\log \gamma(r)}{\log r} \geqq \varlimsup_{\substack{r \rightarrow \infty \\ r \in[0,+\infty)-E}} \frac{\log r(r)}{\log r} .
$$

On the other hand, setting $\int_{E} \frac{d r}{r}=\log \delta<\infty$, for a given $\left\{r_{n}^{\prime}\right\}, r_{n}^{\prime} \in[0, \infty), r_{n}^{\prime} \rightarrow \infty$, there exists a point $r_{n} \in\left[r_{n}^{\prime},(\delta+1) r_{n}^{\prime}\right]-E$. From

$$
\frac{\log \Upsilon\left(r_{n}^{\prime}\right)}{\log r_{n}^{\prime}} \leqq \frac{\log \Upsilon\left(r_{n}\right)}{\log r_{n}^{\prime}} \leqq \frac{\log \Upsilon\left(r_{n}\right)}{\log r_{n}+\log (1 / \delta+1)}=\frac{\log \Upsilon\left(r_{n}\right)}{\log r_{n}(1+o(1))}
$$

it follows that

$$
\varlimsup_{r_{n}^{\prime} \rightarrow \infty} \frac{\log \gamma\left(r_{n}^{\prime}\right)}{\log r_{n}^{\prime}} \leqq \varlimsup_{r_{n \rightarrow \infty}} \frac{\log \gamma\left(r_{n}\right)}{\log r_{n}} \leqq \varlimsup_{\substack{r \rightarrow \infty \\ r \in[0,+\infty)-E}} \frac{\log \gamma(r)}{\log r} .
$$

Since $\left\{r_{n}^{\prime}\right\}$ is arbitrary, we have

$$
\varlimsup_{\substack{r \rightarrow \infty \\ r \in[0,+\infty)}} \frac{\log \gamma(r)}{\log r} \leqq \varlimsup_{\substack{r \rightarrow \infty \\ r \in[0,+\infty)-E}} \frac{\log \gamma(r)}{\log r} .
$$

This proves Lemma 1 .

LEMMA 2. Let $f$ be a solution of the differential equation

$$
f^{(k)}+a_{k-1} f^{(k-1)}+\cdots+a_{0} f=0 \quad(k \geqq 1)
$$

with $a_{0}, \cdots, a_{k-1}$ polynomials. Then $f$ is entire of order

$$
\sigma(f) \leqq 1+\max _{1 \leq \jmath \leq k} \frac{\operatorname{deg} a_{k-\jmath}}{\jmath} .
$$

Proof. see [1].

LEMMA 3. Let $b_{k-\jmath}(\jmath=1, \cdots, k)$ be rational functions having a pole at $\infty$ of order $n_{k-\jmath} \geqq 0, k \geqq 1, f$ be a meromorphic solution of the differential equation

$$
f^{(k)}+b_{k-1} f^{(k-1)}+\cdots+b_{0} f=0
$$

Then, $\sigma(f) \leqq 1+\max _{1 \leq j \leq k} \frac{n_{k-\jmath}}{j}$.

Proof. If $f$ is a rational function, then Lemma 3 holds. Thus, we can now suppose $f$ is a transcendental meromorphic function. If $f$ has a pole at $z_{0}$ of order $\alpha$, and $b_{k-1}, \cdots, b_{0}$ are all analytic at $z_{0}$, then $f^{(k)}+b_{k-1} f^{(k-1)}+\cdots+b_{0} f$ must has a pole at $z_{0}$ of order $a+k$. This contradicts (2.2) and implies that the poles of $f$ can only occur at the poles of $b_{k-\jmath}(j=1, \cdots, k)$. Hence $f$ has only finitely many poles. Now let $f_{1}$ denote the sum of the principal parts of all poles of $f$, then $f_{1}$ is a rational function with $\left|f_{1}\right|=O\left(r^{-1}\right)$, and $f_{2}=f-f_{1}$ is a transcendental entire function. Now substituting $f=f_{1}+f_{2}$ into (2.2), we 
obtain

$$
f_{2}^{(k)}+b_{k-1} f_{2}^{(k-1)}+\cdots+b_{0} f_{2}=-\left(f_{1}^{(k)}+b_{k-1} f_{1}^{(k-1)}+\cdots+b_{0} f_{1}\right)
$$

For sufficiently large $|z|$. we have $b_{k-\jmath}=B_{k-j} z^{n_{k-j}(1+o(1))}\left(B_{k-\jmath} \neq 0\right.$ are constants). Now let $z$ be a point with $|z|=r$ at which $\left|f_{2}(z)\right|=M\left(r, f_{2}\right)$. Since $f_{1}$ is rational, we get

$$
\lim _{r \rightarrow \infty} \frac{f_{1}^{(k)}+b_{k-1} f_{1}^{(k-1)}+\cdots+b_{0} f_{1}}{f_{2}(z)}=\lim _{r \rightarrow \infty} \frac{f_{1}^{(k)}+b_{k-1} f_{1}^{(k-1)}+\cdots+b_{0} f_{1}}{M\left(r, f_{2}\right)}=0 .
$$

From the Wiman-Valiron theory (see [4], [6], [7]), we have basic formulas

$$
\frac{f_{2}^{(\jmath)}(z)}{f_{2}(z)}=\left(\frac{\Upsilon_{f_{2}}(r)}{z}\right)^{\jmath}(1+o(1)), \quad \jmath=1, \cdots, k
$$

where $|z|=r,\left|f_{2}(z)\right|=M\left(r, f_{2}\right), r \in E, \int_{E} \frac{d r}{r}<\infty, \gamma_{f_{2}}(r)$ denotes the centralindex of $f_{2}(z)$. Substituting (2.4), (2.5) into (2.3), we have

$$
\left(\frac{\Upsilon_{f_{2}}(r)}{z}\right)^{k}(1+o(1))+B_{k-1} z^{n_{k-1}}\left(\frac{\Upsilon_{f_{2}}(r)}{z}\right)^{k-1}(1+o(1))+\cdots+B_{0} z^{n_{0}}(1+o(1))=o(1) .
$$

Since the solutions of an algebraic equation are continuous functions in its coefficients (see [4, P. 228]). As $r \rightarrow \infty$, the solutions of (2.6) are asymptotically equal to the solutions of the algebraic equation

$$
\left(\frac{\Upsilon_{f_{2}}(r)}{z}\right)^{k}+B_{k-1} z^{n_{k-1}}\left(\frac{\Upsilon_{f_{2}}(r)}{z}\right)^{k-1}+\cdots+B_{0} z^{n_{0}}=0
$$

The solution $\gamma_{f_{2}}(r)$ of (2.7) is the centralindex of the solution $g$ of the differential equation with polynomial coefficients

$$
g^{(k)}+B_{k-1} z^{n_{k-1}} g^{(k-1)}+\cdots+B_{0} z^{n_{0}} g=0 .
$$

So by Lemma 2 we have $\sigma(g) \leqq 1+\max _{1 \leqq \jmath k} \frac{n_{k-\jmath}}{\jmath}$, and by the Wiman-Valiron theory, we obtain

$$
\begin{gathered}
\sigma(g)=\varlimsup_{r \rightarrow \infty} \frac{\log \gamma_{f_{2}}(r)}{\log r}=\sigma\left(f_{2}\right), \\
\sigma(f)=\sigma\left(f_{1}+f_{2}\right)=\sigma\left(f_{2}\right) \leqq 1+\max _{1 \leqq \jmath \leqq k} \frac{n_{k-\jmath}}{\jmath} .
\end{gathered}
$$

LEMMA 4. Let $A \neq 0, b_{k-\jmath}(j=1, \cdots, k)$ be retional functions, $b_{k-\jmath}$ have a pole at $\infty$ order $n_{k-\jmath} \geqq 0, k \geqq 1, P$ be a polynomial such that $\operatorname{deg} P=\beta$, and $f$ be a meromorphic solution of equation (1.4). Then $f$ has only finitely many poles. If $\sigma(f)>\beta$, then $\sigma(f) \leqq 1+\max _{1 \leqq \jmath \leqq k} \frac{n_{k-\jmath}}{\jmath}$. 
Proof. By the same proof as in the proof of Lemma 3, we see that poles of $f$ can only occur at poles of $b_{k-\jmath}(j=1, \cdots, k)$. Hence $f$ has only fimitely many poles. If $\sigma(f)>\beta$, we let $f_{1}, f_{2}$ denote the same as in the proof of lemma 3. Now substituting $f=f_{1}+f_{2}$ into (1.4), we obtain

$$
f_{2}^{(k)}+b_{k-1} f_{2}^{(k-1)}+\cdots+b_{0} f_{2}=A e^{p}-\left(f_{1}^{(k)}+b_{k-1} f_{1}^{(k-1)}+\cdots+b_{0} f_{1}\right) A e^{p}-C,
$$

where $C=f_{1}^{(k)}+b_{k-1} f_{1}^{(k-1)}+\cdots+b_{0} f_{1}$. Now let $z$ be a point with $|z|=r$ at which $\left|f_{2}(z)\right|=M\left(r, f_{2}\right)$. From the Wiman-Valiron theory (see [4], [6], [7]), (2.5) holds. Now for a given $\varepsilon, 0<3 \varepsilon<\sigma(f)-\beta$, there exists $\left\{r_{n}^{\prime}\right\}\left(r_{n}^{\prime} \rightarrow \infty\right)$ such that $M\left(r_{n}^{\prime}, f_{2}\right)>\exp \left\{r_{n}^{\prime \sigma(f)-\varepsilon}\right\}$. Setting $\int_{E} \frac{d r}{r}=\log \delta<\infty$, there exists a point $r_{n}$ $\in\left[r_{n}^{\prime},(\delta+1) r_{n}^{\prime}\right]-E$. At such points $r_{n}$, we have

$$
M\left(r_{n}, f_{2}\right) \geqq M\left(r_{n}^{\prime}, f_{2}\right)>\exp \left\{r_{n}^{\prime \sigma(f)-\varepsilon}\right\}>\exp \left\{\frac{r_{n}^{\sigma(f)-\varepsilon}}{(\delta+1)^{\sigma(f)}}\right\}>\exp \left\{r_{n}^{\sigma(f)-2 \varepsilon}\right\} .
$$

In addition for sufficiently large $r_{n}$ we have

$$
\left|A e^{p}-C\right| \leqq \exp \left\{r_{n}^{\beta+\varepsilon}\right\} .
$$

So

$$
\left|\frac{A e^{p}-C}{M\left(r_{n}, f_{2}\right)}\right| \leqq \exp \left\{r_{n}^{\beta+\varepsilon}-r_{n}^{\sigma(f)-2 \varepsilon}\right\} \longrightarrow 0 \quad\left(r_{n} \rightarrow \infty\right)
$$

Therefore, at such points $\left|z_{n}\right|=r_{n}\left(r_{n} \equiv E,\left|f_{2}\left(z_{n}\right)\right|=M\left(r_{n}, f_{2}\right)\right)$, substituting (2.5) into (2.8), we have

$$
\left(\frac{\Upsilon_{f_{2}}\left(r_{n}\right)}{z_{n}}\right)^{k}(1+o(1))+B_{k-1} z_{n}^{n k-1}\left(\frac{\Upsilon_{f_{2}}\left(r_{n}\right)}{z_{n}}\right)^{k-1}(1+o(1))+\cdots+B_{0} z_{n}^{n_{0}}(1+o(1))=o(1)
$$

As $r_{n} \rightarrow \infty$, at the points $r_{n}$, a solution $r_{f_{2}}\left(r_{n}\right)$ of (2.9) is asymptotically equal to a solution of an algebraic equation

$$
\left(\frac{\Upsilon_{f_{2}}\left(r_{n}\right)}{z_{n}}\right)^{k}+B_{k-1} z_{n}^{n k-1}\left(\frac{\Upsilon_{f_{2}}\left(r_{n}\right)}{z_{n}}\right)^{k-1}+\cdots+B_{0} z_{n}^{n_{0}}=0
$$

Thus

$$
r_{f_{2}}\left(r_{n}\right) \sim c_{1} r_{n}^{\alpha_{1}}
$$

where $c_{1} \neq 0$ is a constant, the possible values of $\alpha_{1}$ should coincide with the possible orders of growth of transcendental solutions of equation

$$
f_{2}^{(k)}+B_{k-1} z^{n_{k-1}} f_{2}^{(k-1)}+\cdots+B_{0} z^{n_{0}} f_{2}=0 .
$$

But Lemma 3 gives $a_{1} \leqq 1+\max _{1 \leqq \jmath \leqq k} \frac{n_{k-\jmath}}{j}$.

On the other hand, differentiating (2.8) gives 


$$
\begin{aligned}
& f_{2}^{(k+1)}+b_{k-1} f_{2}^{(k)}+\left(b_{k-1}^{\prime}+b_{k-2}\right) f_{2}^{(k-1)}+\cdots+b_{0}^{\prime} f_{2}=A^{\prime} e^{p}+A p^{\prime} e^{p}-C^{\prime} \\
&=\left(\frac{A^{\prime}}{A}+p^{\prime}\right)\left(A e^{p}-C\right)+C\left(\frac{A^{\prime}}{A}+p^{\prime}\right)-C^{\prime} \\
&=\left(\frac{A^{\prime}}{A}+p^{\prime}\right)\left(f_{2}^{(k)}+b_{k-1} f_{2}^{(k-1)}+\cdots+b_{0} f_{2}\right)+C\left(\frac{A^{\prime}}{A}+p^{\prime}\right)-C^{\prime}, \\
& f_{2}^{(k+1)}+ {\left[b_{k-1}-\left(\frac{A^{\prime}}{A}+p^{\prime}\right)\right] f_{2}^{(k)}+\left[b_{k-1}^{\prime}+b_{k-2}-\left(\frac{A^{\prime}}{A}+p^{\prime}\right) b_{k-1}\right] f_{2}^{(k-1)}+} \\
& \cdots+\left[b_{0}^{\prime}-\left(\frac{A^{\prime}}{A}+p^{\prime}\right) b_{0}\right] f_{2}=C\left(\frac{A^{\prime}}{A}+p^{\prime}\right)-C^{\prime},
\end{aligned}
$$

i. e. $f_{2}$ also solves a linear differential equation with rational coefficients, since $f_{2}$ is a transcendental entire function, by the reasoning in [6, P. 106-108], for sufficiently larger $r$ we have $r_{f_{2}}(r) \sim c_{2} r^{\alpha_{2}}(r \in E)$, with $c_{2}$ a constant, $\alpha_{2}$ a rational number. But by (2.11), we have $c_{1} r_{n}^{\alpha_{1}} \sim c_{2} r_{n}^{\alpha_{2}}$. So $c_{1}=c_{2}, \alpha_{1}=\alpha_{2}$ must hold. And by Lemma 1 we get $\sigma\left(f_{2}\right)=\alpha_{2} \leqq 1+\max _{1 \leqq \jmath \leqq} \frac{n_{k-\jmath}}{\jmath}$. Therefore, $\sigma(f)=\sigma\left(f_{2}\right) \leqq 1+\max _{1 \leqq \jmath \leqq k} \frac{n_{k-\jmath}}{\jmath}$.

LEMMA 5. Let $b_{k-\jmath}(\jmath=1, \cdots, k)$ be rational functions having a pole at $\infty$ of order $n_{k-\jmath} \geqq 0, k \geqq 1, B(z)$ be a meromorphic function with $\sigma(B)=\beta$ satısfying (1.3). If all solutions of the differential equation (1.5) are meromorphic functions, then $\sigma(f)=\beta$.

Proof. It is easy to see that $\sigma(f) \geqq \sigma(B)=\beta$ from (1.5). On the other hand, all solutions of (2.2) that is the corresponding homogeneous differential equation of (1.5) are meromorphic functions, we assume that $\left\{f_{1}, \cdots, f_{k}\right\}$ is a fundamental solution set of (2.2). By Lemma 3 we have $\sigma\left(f_{\imath}\right) \leqq 1+\max _{1 \leqq \jmath \leqq k} \frac{n_{k-\jmath}}{\jmath}\left(_{\imath}=\right.$ $1, \cdots, k)$.

By variation of parameters, we can write

$$
f=A_{1}(z) f_{1}+\cdots+A_{k}(z) f_{k},
$$

where $A_{1}(z), \cdots, A_{k}(z)$ are determined by

$$
\begin{aligned}
& A_{1}^{\prime} f_{1}+\cdots+A_{k}^{\prime} f_{k}=0 \\
& A_{1}^{\prime} f_{1}^{\prime}+\cdots+A_{k}^{\prime} f_{k}^{\prime}=0 \\
& \cdots \cdots \cdots \cdots \\
& A_{1}^{\prime} f_{1}^{(k-1)}+\cdots+A_{k}^{\prime} f_{k}^{(k-1)}=B .
\end{aligned}
$$

Noting that the Wronskian $W\left(f_{1}, \cdots, f_{k}\right)$ is a differential polynomial in $f_{1}, \cdots, f_{k}$ with constant coefficients, it is easy to know that $\sigma(w) \leqq 1+\max _{1 \leqq j \leqq k} \frac{n_{k-1}}{\jmath}$. Set 


$$
w_{\imath}=\left|\begin{array}{cc}
f_{1}, \cdots \cdots, & 0, \cdots, f_{k} \\
\vdots & \vdots \\
\dot{f}_{1}^{(k-1)}, \cdots, & B, \cdots,
\end{array}\right|=B \cdot g_{\imath}, \quad i=1, \cdots, k,
$$

where $g_{\imath}$ are differential polynomials in $f_{1}, \cdots, f_{k}$ with constant coefficients. So $\sigma\left(g_{\imath}\right) \leqq 1+\max _{1 \leq \jmath \leq k} \frac{n_{k-\jmath}}{j}$ also hold. Since $A_{i}^{\prime}=\frac{W_{\imath}}{W}=\frac{B g_{\imath}}{W}$ and $\sigma(B)=\beta>1+\max _{1 \leq \jmath \leq k} \frac{n_{k-\jmath}}{j}$, we have $\sigma\left(A_{j}^{\prime}\right) \leqq \beta, \sigma\left(A_{\imath}\right)=\sigma\left(A_{\imath}^{\prime}\right) \leqq \beta$. And from (2.12) we get $\sigma(f) \leqq \beta$ Hence $\sigma(f)=\beta$ must hold. (It is not difficult to see that we can suppose all $A_{\imath}$ are meromorphic functions here.)

LEMMA 6. Let $U \equiv 0$ be a meromorphic function with $\sigma(U)<\infty, b_{k-\jmath}(j=$ $1, \cdots, k)$ be rational functions. If $f$ is a meromorphic solution of the differential equation

$$
f^{(k)}+b_{k-1} f^{(k-1)}+\cdots+b_{\mathrm{o}} f=U
$$

such that $\sigma(U)<\sigma(f)<\infty$, then $\bar{\lambda}(f)=\lambda(f)=\sigma(f)$.

Proof. We can write from (2.13)

$$
\frac{1}{f}=\frac{1}{U}\left(\frac{f^{(k)}}{f}+b_{k-1} \frac{f^{(k-1)}}{f}+\cdots+b_{0}\right) .
$$

Since $\sigma(f)<\infty$, we have $m\left(r,\left(f^{(j)} / f\right)\right)=O(\log r)(j=1, \cdots, k)$, thus,

$$
\begin{aligned}
m\left(r, \frac{1}{f}\right) & \leqq m\left(r, \frac{1}{U}\right)+m\left(r, \frac{f^{(k)}}{f}\right)+\cdots+m\left(r, \frac{f^{\prime}}{f}\right)+O(\log r) \\
& =m\left(r, \frac{1}{U}\right)+O(\log r) .
\end{aligned}
$$

Because $b_{k-1}, \cdots, b_{0}$ are rational functions, $b_{k-1}, \cdots, b_{0}$ must be analytic at $z_{0}$ as $\left|z_{0}\right|$ is sufficiently large. If $f$ has a zero at $z_{0}$ of order $\beta(>k)$, then $U$ must have a zero at $z_{0}$ of order $\beta-k$. Hence,

and

$$
n\left(r, \frac{1}{f}\right) \leqq k \bar{n}\left(r, \frac{1}{f}\right)+n\left(r, \frac{1}{U}\right)+O(1),
$$

$$
N\left(r, \frac{1}{f}\right) \leqq k \bar{N}\left(r, \frac{1}{f}\right)+N\left(r, \frac{1}{U}\right)+O(\log r) .
$$

(2.15) and (2.16) give

$$
\begin{aligned}
T(r, f) & =T\left(r, \frac{1}{f}\right)+O(1) \leqq k \bar{N}\left(r, \frac{1}{f}\right)+T\left(r, \frac{1}{U}\right)+O(\log r) \\
& =k \bar{N}\left(r, \frac{1}{f}\right)+T(r, U)+O(\log r) .
\end{aligned}
$$

Setting $\sigma(f)=\alpha>\sigma(U)$, there exists $\left\{r_{n}\right\} \quad\left(r_{n} \rightarrow \infty\right)$ such that 


$$
\lim _{r_{n} \rightarrow \infty} \frac{\log T\left(r_{n}, f\right)}{\log r}=\alpha .
$$

For a given $\varepsilon$ with $0<2 \varepsilon<\alpha-\sigma(U)$, as $r_{n}$ is sufficiently large, we have

Therefore

$$
T\left(r_{n}, f\right)>r_{n}^{\alpha-\varepsilon}, \quad T\left(r_{n}, U\right)<r_{n}^{\sigma(U)+\varepsilon} .
$$

and

$$
\frac{T\left(r_{n}, U\right)}{T\left(r_{n}, f\right)}<r_{n}^{2 \varepsilon-(\alpha-\sigma(U))} \longrightarrow 0 \quad\left(r_{n} \rightarrow \infty\right)
$$

$$
T\left(r_{n}, U\right) \leqq \frac{1}{2} T\left(r_{n}, f\right)
$$

holds for sufficiently $r_{n}$. From (2.17) we obtain

$$
T\left(r_{n}, f\right) \leqq 2 k \bar{N}\left(r_{n}, \frac{1}{f}\right)+O\left(\log r_{n}\right)
$$

for such $r_{n}$. Thus,

$$
\sigma(f)=\alpha=\lim _{r_{n} \rightarrow \infty} \frac{\log T\left(r_{n}, f\right)}{\log r_{n}} \leqq \varlimsup_{r_{n \rightarrow \infty}} \frac{\log \bar{N}\left(r_{n},(1 / f)\right)}{\log r_{n}} \leqq \bar{\lambda}(f) .
$$

So we get $\bar{\lambda}(f)=\lambda(f)=\sigma(f)$.

LEMMA 7. Let $b_{k-\jmath}(\jmath=1, \cdots, k)$ be rational functions having a pole at $\infty$ of order $n_{k-\jmath} \geqq 0, k \geqq 1, B \neq 0$ be a meromorphic function with $\sigma(B)=\beta<\infty$. If all solutions of the differential equation (1.5) are meromorphic functions, then $\lambda(1 / f)=\lambda(1 / B), \bar{\lambda}(1 / f)=\bar{\lambda}(1 / B)$,

$$
\max \left\{\lambda(f), \lambda\left(\frac{1}{f}\right)\right\} \geqq \max \left\{\lambda(B), \lambda\left(\frac{1}{B}\right)\right\} .
$$

Proof. Since $b_{k-\jmath}(\jmath=1, \cdots, k)$ have only finitely many poles, and as $b_{k-1}, \cdots, b_{0}$ are all analytic at $z_{0}, f$ has a pole at $z_{0}$ of order $\alpha$ if and only if $B$ has a pole at $z_{0}$ of order $\alpha+k$, we have $\bar{\lambda}(1 / f)=\bar{\lambda}(1 / B)$. From $(\alpha+k / 2 k)=$ $(\alpha / 2 k)+(1 / 2) \leqq \alpha$, it follows that

$$
\frac{1}{2 k} n(r, B)+O(1) \leqq n(r, f) \leqq n(r, B)+O(1),
$$

and

$$
-\frac{1}{2 k} N(r, B)+O(\log r) \leqq N(r, f) \leqq N(r, B)+O(\log r) .
$$

Therefore, $\lambda(1 / f)=\lambda(1 / B)$,

By the proof of Lemma 5 we know that $\sigma(f)<\infty$. So we can write

$$
f=z^{m_{1}} \frac{H_{1}}{Q_{1}} e^{P_{1}}, \quad B=z^{m_{2}} \frac{H_{2}}{Q_{2}} e^{P_{2}},
$$


where $m_{1}, m_{2}$ are integers, $H_{1}$ and $H_{2}$ are canonical products formed respectively with the nonzero zeros of $f$ and $B, Q_{1}$ and $Q_{2}$ are canonical products formed respectively with the nonzero poles of $f$ and $B, P_{1}$ and $P_{2}$ are polynomials with $\operatorname{deg} P_{1} \leqq \sigma(f) . \operatorname{deg} P_{2} \leqq \sigma(B)$. Substituting (2.19) into (1.5) we have

$$
F\left(H_{1}, Q_{1}\right)=z^{m_{2}} \frac{H_{2}}{Q_{2}} e^{P_{2}-P_{1}},
$$

where $F$ is a rational function in $H_{1}, Q_{1}$ and $H_{1}^{(\jmath)}, Q_{1}^{(j)}(\jmath=1, \cdots, k)$ with polynomial coefficients. From (2.20), we get

$$
\max \left\{\sigma\left(H_{1}\right), \sigma\left(Q_{1}\right)\right\} \geqq \sigma(F)=\sigma\left(z^{m_{2}} \frac{H_{2}}{Q_{2}} e^{P_{2}-P_{1}}\right) \geqq \max \left\{\sigma\left(H_{2}\right), \sigma\left(Q_{2}\right)\right\} .
$$

So (2.18) holds.

LEMMA 8. Let $\beta$ be a positive integer and $\beta>1, b_{k-\jmath}(\jmath=1, \cdots, k)$ be rational functions having a pole at $\infty$ of order $j(\beta-1), k \geqq 1, g$ be a meromorphic solution of the homogeneous differentıal equation

$$
g^{(k)}+b_{k-1} g^{(k-1)}+\cdots+b_{0} g=0 .
$$

Then $\sigma(g)=\beta$.

Proof. Using the same proof as in the proof of Lemma 3, we see that $g$ has only finitely many poles. Now let $g_{1}$ denote the sum of the principal parts of all poles of $g$. Then $g_{1}$ is a rational function with $\left|g_{1}\right|=o\left(r^{-1}\right)$, and $g_{2}=$ $g-g_{1}$ is an entire function. Substituting $g=g_{1}+g_{2}$ into (2.21), we obtain

$$
g_{2}^{(k)}+b_{k-1} g_{2}^{(k-1)}+\cdots+b_{0} g_{2}=-\left(g_{1}^{(k)}+b_{k-1} g_{1}^{(k-1)}+\cdots+b_{0} g_{1}\right) .
$$

If $g_{2}$ is a polynomial, then there is only one term $b_{0} g_{2}$ with degree $k(\beta-1)+$ $\operatorname{deg} g_{2}$ being the highest one in (2.22). This is impossible. So $g_{2}$ must be a transcendental entire function. Now, we use the same proof as in the proof of Lemma 3. Let $z$ be a point with $|z|=r$ at which $\left|g_{2}(z)\right|=M\left(r, g_{2}\right), \Upsilon_{g_{2}}(r)$ denote the centralindex of $f_{2}(z), E \subset[0, \infty)$ be a set such that $\int_{E} \frac{d r}{r}<\infty$.

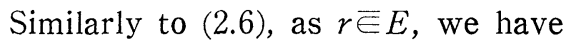

$$
\left(\frac{\Upsilon_{g_{2}}(r)}{z}\right)^{k}(1+o(1))+B_{k-1} z^{\beta-1}\left(\frac{\Upsilon_{g_{2}}(r)}{z}\right)^{k-1}(1+o(1))+\cdots+B_{0} z^{k(\beta-1)}(1+o(1))=o(1) .
$$

Set $\sigma\left(g_{2}\right)=\alpha$. Then by the reasoning in [6, P. 106-108] we have $\Upsilon_{g_{2}}(r) \sim c z^{\alpha}$ $(|z|=r \bar{E} E, \quad c \neq 0$ is a constant) as $r \rightarrow \infty$. Substituting it into (2.24), it is easy to see that the degrees of all terms of (2.24) are respectively

$$
k(\alpha-1), j(\beta-1)+(k-j)(\alpha-1) \quad(\jmath=1, \cdots, k-1), k(\beta-1) .
$$

From the Wilman-Valiron theory we see that $\alpha=\beta$ is the only possible value. 
Therefore, by Lemma 1 we get $\sigma\left(g_{2}\right)=\beta$, and $\sigma(g)=\sigma\left(g_{2}\right)=\beta$.

Lemma 9. Let $\beta, b_{k-\jmath}(j=1, \cdots, k)$ be the same as Lemma $8, A \neq 0$ be a rational function having $a$ pole at $\infty$ of order $n_{A}$ consider the differential equation

$$
g^{(k)}+b_{k-1} g^{(k-1)}+\cdots+b_{0} g=A .
$$

If $n_{A}<k(\beta-1)$, then every meromorphic solution $g$ of $(2.25)$ is of $\sigma(g)=\beta$. If $n_{A} \geqq k(\beta-1)$, then all meromorphic solutions of (2.25) satisfy $\sigma(g)=\beta$ except at most one possible. The possible exceptional one $g_{0}$ is a rational function.

Proof. Assume $g$ is a meromorphic solution of (2.25). Clearly, $g$ has only finitely many poles. Let $g_{1}$ denote the sum of the principal parts of all poles of $g$. Then $g_{1}$ is a rational function, and $g_{2}=g-g_{1}$ is an entire function. Now substituting $g=g_{1}+g_{2}$ into (2.25), we get

$$
g_{2}^{(k)}+b_{k-1} g_{2}^{(k-1)}+\cdots+b_{0} g_{2}=A-\left(g_{1}^{(k)}+b_{k-1} g_{1}^{(k-1)}+\cdots+b_{0} g_{1}\right)
$$

Divide the discussion into two cases. Case I. $n_{A} \geqq k(\beta-1)$. In this case, if $g_{2}$ is a polynomial solution, thus $g_{0}=g_{1}+g_{2}$ is a rational solution of (2.25). If $g_{2}$ is a transcendental entitre function, we can use the same proof as in Lemma 8 to get $\sigma(g)=\sigma\left(g_{1}+g_{2}\right)=\sigma\left(g_{2}\right)=\beta$.

Case II. $n_{A}<k(\beta-1)$. In this case, if $g_{2}$ is a polynomial, then there is only one term $b_{0} g_{2}$ with degree $k(\beta-1)+\operatorname{deg} g_{2}$ being the highest one in (2.26). This is impossible. Therefore, $g_{2}$ is a transcendental entitre function. Using the same proof as in Lemma 8, we can get $\sigma(g)=\sigma\left(g_{1}+g_{2}\right)=\sigma\left(g_{2}\right)=\beta$.

We affirm that equation (2.25) can only possess at most one exceptional rational solution $g_{0}$. In fact, if $\bar{g}_{0}$ is the other one, then $\sigma\left(g_{0}-\bar{g}_{0}\right)<\beta$. But $g_{0}-\bar{g}_{0}$ is a solution of the corresponding homogeneous equation (2.21) of (2.25). This contradicts Lemma 8.

LEMMA 10. Let $\beta, b_{k-\jmath}(j=1, \cdots, k)$ be the same as Lemma $8, U \neq 0$ be a meromorphic function with $\sigma(U)<\beta$, If all solutions of the equation

$$
g^{(k)}+b_{k-1} g^{(k-1)}+\cdots+b_{0} g=U
$$

are meromorphic functions, then all solutions of (2.27) satisfy $\sigma(g)=\beta$ except at most one possible. The possible exceptional one $\bar{g}$ is of $\sigma(\bar{g})<\beta$.

Proof. Assume that $\left\{g_{1}, \cdots, g_{k}\right\}$ is a fundamental solution set of (2.21) that is the corresponding homogeneous differential equation of (2.27). By Lemma 8 , we have $\sigma\left(g_{j}\right)=\beta(j=1, \cdots, k)$.

Using the method of variation of parameters as in Lemma 5 , we can prove that all solutions of $(2.27)$ are of $\sigma(g) \leqq \beta$.

Using the same proof as in the proof of Lemma 9, we see that (2.27) possesses at most one exceptional solution $\bar{g}$ of order $\sigma(\bar{g})<\beta$, the other solutions 
$g$ are all of order $\sigma(g)=\beta$.

\section{$\S 3$. Proof of Theorems.}

Proof of Theorem 1. (a) By (1.4) we have $\sigma(f) \geqq \sigma\left(A e^{p}\right)=\beta$. If $\sigma(f)>\beta$, from Lemma 4 we have $\sigma(f) \leqq 1+\max _{1 \leqq \jmath \leqq k} \frac{n_{k-\jmath}}{\jmath}$. This is a contradiction. Therefore $\sigma(f)=\beta$. And $f$ has only finitely many poles from Lemma 4 .

(b) Set $f=g e^{p}$. Then $\lambda(f)=\lambda(g), \bar{\lambda}(f)=\bar{\lambda}(g)$. Substituting $f=g e^{p}$ into (1.4), we have

$$
g^{(k)} d_{k-1} g^{(k-1)}+\cdots+d_{0} g=A,
$$

where $d_{k-1}, \cdots, d_{0}$ are rational functions. To work out $\sigma(g)$, we need $d_{k-\jmath}$ $(\jmath=1, \cdots, k)$ in more detailed form. It is easy to check by induction that we have for $m \geqq 2$ (see [5])

$$
f^{(m)}=\left\{g^{(m)}+m p^{\prime} g^{(m-1)}+\sum_{\imath=2}^{m}\left[c_{m}^{\imath}\left(p^{\prime}\right)^{\imath}+H_{\imath-1}\left(p^{\prime}\right)\right] g^{(m-i)}\right\} e^{p},
$$

where $H_{\imath-1}\left(p^{\prime}\right)$ are differential polynomials in $p^{\prime}$ and its derivatives of total degree $i-1$ with constant coefficients. It is easy to see that the derivatives of $H_{\imath-1}\left(p^{\prime}\right)$ as to $z$ are of the same form $H_{\imath-1}\left(p^{\prime}\right) \cdot C_{m}^{\imath}$ is the usual notation for the binomial coefficients. (1.4) and (3.2) give

$$
\left\{\begin{aligned}
d_{k-\jmath}= & b_{k-j}+(k-j-i) b_{k-\jmath+1} p^{\prime}+\sum_{\imath=2}^{\jmath} b_{k-\jmath+i}\left(C_{k-\jmath+i}^{\imath}\left(p^{\prime}\right)^{\imath}+H_{\imath-1}\left(p^{\prime}\right)\right) \\
d_{k-1}= & \left(\jmath=2, \cdots, k, b_{k-1} \equiv 1\right), k p^{\prime} .
\end{aligned}\right.
$$

Since $\beta>1+\max _{1 \leqq \jmath \leq k} \frac{n_{k-\jmath}}{\jmath}$, the degree $\jmath(\beta-1)$ of the term $b_{k} C_{k}^{\jmath}\left(p^{\prime}\right)^{\jmath}=C_{k}^{\jmath}\left(p^{\prime}\right)^{\jmath} \quad(\imath=j)$ is the highest one in the first equality of (3.3). Hence $d_{k-3}$, must have a pole at $\infty$ of order $j(\beta-1)$. If $n_{A} \geqq k(\beta-1)$, then from Lemma $9,(3.1)$ may have one exceptional rational solution $A_{0}$ the other meromorphic solutions are all of $\sigma(g)=\beta$. By Lemma 6 we have $\bar{\lambda}(g)=\lambda(g)=\sigma(g)=\beta$. Therefore (1.4) may have one exceptional solution $f_{0}=A_{0} e^{p}$ ( $A_{0}$ is a rational function), the other meromorphic solutions $f=g e^{p}$ are all of $\bar{\lambda}(f)=\lambda(f)=\sigma(f)=\bar{\lambda}(g)=\beta$. If $n_{A}<k(\beta-1)$, then from Lemma 9 and Lemma 6, all meromorphic solutions $g$ of (3.1) are of $\bar{\lambda}(g)$ $=\lambda(g)=\sigma(g)=\beta$. Therefore all meromorphic solutions $f=g e^{p}$ of (1.4) are of

$$
\bar{\lambda}(f)=\lambda(f)=\sigma(f)=\beta .
$$

Proof of Theorem 2. (a) It is easy to see that $\sigma(f) \geqq \beta$ from (1.4). If $\sigma(f)>\beta$, then $\sigma(f) \leqq 1+\max _{1 \leq j \leq k} \frac{n_{k-\jmath}}{j}$ from Lemma 4 . And by Lemma $4, f$ has only finitely many poles. 
(b) If $\sigma(f)>\beta$, then $\bar{\lambda}(f)=\lambda(f)=\sigma(f)$ from Lemma 6 .

Proof of Theorem 3. (a) From Lemma 5, we have $\sigma\{f\}=\beta$.

(b) By Lemma 7 , we see that $\lambda(1 / f)=\lambda(1 / B), \bar{\lambda}(1 / f)=\bar{\lambda}(1 / B)$. If $\lambda(B)>$ $\lambda(1 / B)$, we have $\lambda(f) \geqq \lambda(B)$ by Lemma 7 .

(c) If $\beta>\max \{\lambda(B), \lambda(1 / B)\}$ we can write

$$
B=z^{m} \frac{H}{Q} e^{p(z)}=U e^{p(z)},
$$

where $m$ is an integer, $H$ and $Q$ are canonical products formed respectively with the nonzero zeros and nonzero poles of $B, U=z^{m}(H / Q), \sigma(U)<\beta$, then $\beta$ must be an integer, $p(z)$ is a polynomial with $\operatorname{deg} P=\beta$.

We set $f=g e^{p}$. Then $\lambda(g)=\lambda(f), \bar{\lambda}(g)=\bar{\lambda}(f)$. Substituting $f=g e^{p}$ into (1.5), we have

$$
g^{(k)}+d_{k-1} g^{(k-1)}+\cdots+d_{0} g=U
$$

where $d_{k-1}, \cdots, d_{0}$ are retional functions.

Using the same proof as in the proof of Theorem 1 (b), we see that $d_{k-1}$ must have a pole at $\infty$ of order $j(\beta-1)$. Hence from Lemma 10, we see that all meromorphic solutions of (3.4) satisfy $\sigma(g)=\beta$ except at most one possible. The possible exceptional one $\bar{g}$ is of $\sigma(\bar{g})<\beta$. If $\sigma(\bar{g})<\beta$, then $\lambda(\bar{g})<\beta$. If $\sigma(g)=\beta$, by $\sigma(U)<\beta$ and Lemma 6 , we have $\bar{\lambda}(g)=\lambda(g)=\sigma(g)=\beta$. Therefore the equation (1.5) may have at most one exceptional solution $f_{0}=\bar{g} e^{p}$ with $\lambda\left(f_{0}\right)$ $=\lambda(\bar{g})<\beta$, the other meromorphic solutions $f=g e^{p}$ of (1.5) are all of $\bar{\lambda}(f)=\lambda(f)$ $=\bar{\lambda}(g)=\beta$.

Proof of Theorem 4. (a) By (1.5), we have $\sigma(f) \geqq \beta$. On the other hand, since all solutions of (1.5) are meromorphic functions, all solutions of (2.2) that is the corresponding homogeneous equation of (1.5) are meromorphic functions. Assume $\left\{f_{1}, \cdots, f_{k}\right\}$ is fundamental solution set of (2.2). By Lemma 3 we have $\sigma\left(f_{\imath}\right) \leqq 1+\max _{1 \leqq \jmath \leqq k} \frac{n_{k-\jmath}}{j}(i=1, \cdots, k)$.

By variation of parametes, for a solution $f$ of (1.5), we can write

$$
f=A_{1}(z) f_{1}+\cdots+A_{k}(z) f_{k} .
$$

Using the same proof as in the proof of Lemma 5 and noting that $\beta \leqq 1+$ $\max _{1 \leqq \jmath \leqq k} \frac{n_{k-\jmath}}{j}$, we have $\sigma\left(A_{j}\right)=\sigma\left(A_{j}^{\prime}\right) \leqq 1+\max _{1 \leqq j \leqq k} \frac{n_{k-\jmath}}{j}$. Therefore $\beta \leqq \sigma(f) \leqq 1+\max _{1 \leqq \jmath \leqq k} \frac{n_{k-\jmath}}{\jmath}$.

(b) By Lemma 7 we have $\lambda(1 / f)=\lambda(1 / B), \bar{\lambda}(1 / f)=\bar{\lambda}(1 / B), \max \{\lambda(f), \lambda(1 / f)\}$ $\geqq \max \{\lambda(B), \lambda(1 / B)\}$. Therefore, $\lambda(f) \geqq \lambda(B)$, if $\lambda(B)=\lambda(1 / B)$.

(c) If $\sigma(f)>\sigma(B)$, then by Lemma 6 we have $\bar{\lambda}(f)=\lambda(f)=\sigma(f)$. 


\section{$\S 4$. Examples for the exceptional solution.}

Example 1. (concerning the exceptional solution in Theorem 1)

$f_{0}=e f^{z^{3}}$ solves $f^{\prime \prime}+z f^{\prime}+z^{2} f=\left(9 z^{4}+3 z^{3}+z^{2}+6 z\right) e^{z^{3}}$. There $1+\max _{1 \leqq \jmath \leqq k} \frac{n_{k-\jmath}}{j}=2$, $\beta=3, \operatorname{deg} A=4 \geqq k(\beta-1)$. And $f_{0}$ satisfies that $\lambda\left(f_{0}\right)=0<\sigma\left(f_{0}\right)=3=\sigma\left(A e^{p}\right)$.

Example 2. (concerning the exceptional solution in Theorem 3)

$f_{0}=\sin z \cdot e^{z^{2}}$ is an exceptional solution of $f^{\prime \prime}-f=\left(4 z \cos z+4 z^{2} \sin z\right) e^{z^{2}}$. There $1+\max _{1 \leqq \jmath \leq k} \frac{n_{k-\jmath}}{\jmath}=1, \beta=\sigma(B)=2, \beta>\max \{\lambda(B), \lambda(1 / B)\}$. And $f_{0}$ satisfies $\sigma\left(f_{0}\right)=2$, $\lambda\left(f_{0}\right)=1<\beta$.

Acknowledgement. The authors would like to thank the referee for valuable suggestions to improve our paper.

\section{REFERENCES}

[1] G. Frank And S. Hellerstein, On the meromorphic solutions of non-homogeneous linear differential equations with polynomial coefficients, Proc London Math Soc (3), 53 (1986), 407-428.

[2] GAO SHI-AN, On the complex oscillation of solutions of non-homogeneous linear differential equations with polynomial coefficients, Comment. Math. Univ. Sancti Pauli, 38 (1989), No. 1, 11-20.

[3] W. Hayman, Meromorphic Functions, Clarendon Press, Oxford, 1964.

[4] He Yu-zan and Xiao Xiu-zhi, Algebroid Functions and Ordinary Differential Equations, Science Press, 1988 (in Chinese).

[5] I. LAINE, A note on the complex oscillation theory of non-homogeneous linear differential equations, to appear in Comment. Math. Univ. Sancti Pauli.

[6] G. VALiRon, Lectures on the general theory of integral functions, Clelsea, New York, 1949.

[7] G. Valiron, Functions analytiques, Presses Universitaires de France, Paris, 1954.

\section{Chen ZONG-XUAN}

Department of Mathematics,

Nanchang Vocational and Technical Teachers'

College, Nanchang, P.R. China

GAO SHI-AN

Department of MATHEMAtics,

South China Normal University,

Guangzhou, P. R. China 